\title{
Localization of gravity in brane world with arbitrary extra dimensions
}

\author{
A. M. Yazdani ${ }^{1}$, K. Atazadeh ${ }^{2}$ and S. Jalalzadeh ${ }^{2 *}$ \\ ${ }^{2}$ Department of Physics, Shahid Beheshti University G. C., Evin, Tehran 19839, Iran \\ ${ }^{1}$ Department of Physics, Qom Branch, Islamic Azad University, Qom, Iran
}

November 9, 2018

\begin{abstract}
We study the induced 4-dimensional linearized Einstein field equations in an $m$-dimensional bulk space by means of a confining potential. We used the confining potential in this model to localized gravitons on the brane. It is shown that in this approach the mass of graviton is quantized. The cosmological constant problem is also addressed within the context of this approach. We show that the difference between the values of the cosmological constant in particle physics and cosmology stems from our measurements in two different scales, small and large.

pacs: $04.50 .+\mathrm{h}, 04.30 .-\mathrm{w}$
\end{abstract}

\section{Introduction}

The view that our universe might actually have dimensions more than four is anchored on the recent developments in string and M-theories in which gravity arises as a truly higher dimensional. Only in the low energy limit it manifests in the familiar 4-dimensional general relativity. Recent observations have indicated that the cosmological constant $\Lambda$ is a sufficiently small positive value, $\Lambda \sim\left(10^{-3} \mathrm{eV}\right)^{4}$ 1. Consequently it is natural to assume that we live in de a Sitter universe. However it is difficult to explain the reason for the smallness of $\Lambda$, the so called cosmological constant problem. By using the warped extra dimension scenario in [2, new suggestion for the cosmological constant problem was proposed. Randall and Sundrum investigated that the gravity can be localized on flat 3-brane embedded in $A d S_{5}$, the usual 4-dimensional gravity can be reproduced at large distance scale. This is because a normalizable zero mode exists and the effective four-dimensional Planck scale is finite. Due to fine-tuning between the bulk cosmological constant and brane tension, the four-dimensional cosmological constant is vanishing. Furthermore, the close relation between $A d S / C F T$ correspondence and the Randall-Sundrum model is explored in the last couple of years 3, 4, 5. Moreover it is expected that the warped braneworlds provide a new scenario in the framework of phenomenological model building and cosmology. Based on the assumption that physics in four dimensions should be explained by higher dimensional theory, the warped braneworlds with an infinite extra dimension are remarkable scenarios in the field of particle physics.

There are three usual models to consider when studying the dynamics of brane-worlds models featuring boundary terms in the action and sometimes mirror symmetry, such that bulk gravitational waves interfere with the brane-world motion. The first model usually comes together with junction conditions producing an algebraic relationship between the extrinsic curvature and the confined matter [6]. In this model the problem of localization of gravity is discussed in several papers. For example, in [7, the authors addressed the localization of gravity in a FRW type brane embedded in either $A d S_{5}$ or $d S_{5}$ bulk space, and show that the graviton zero mode is trapped on the brane. Non-trapped, massive Kaluza-Klein (KK) modes correspond to a correction to Newton's law. Also in 8 they consider Randall-Sundrum model with localized gravity, replacing the extra compact space-like dimension by a time-like one. In this way they show that the solution to the hierarchy problem can be reconciled with the correct cosmological expansion of the visible universe. One the other hand, In [9], the authors show that, in brane models with a compact extra dimension like the Randall-Sundrum's two

*email: s-jalalzadeh@sbu.ac.ir 
brane model or models with universal extra dimension [10], which allow a zero mode known from KK theories, the five dimensional linearly perturbed Einstein equations are in conflict with observations.

The second model is against the Israel junction conditions for confining matter or gauge fields on the brane by using $Z_{2}$ symmetry. This model was introduced in [11] where matter fields are trapped on a 4-dimensional hypersurface by the action of the confining potential $\mathcal{V}$. In this model, without using $Z_{2}$ symmetry or without postulating any junction condition, Friedman equation is modified by a geometrical term which is defined in terms of the extrinsic curvature, leading to a geometrical interpretation for dark energy and to find a richer set of cosmological solutions in accordance with the current observations [12, 13. In this direction, in [14 the authors developed a brane model, in which the number of non-compact extra dimensions is arbitrary also the dynamics of test particles confined to a brane by the action of such potential at the classical and quantum levels were studied in 14]. In [15, a brane-world model was studied in which matter is confined to the brane through the action of such a potential without using any junction conditions, offering a geometrical explanation for the accelerated expansion of the universe.

Third model for localizing gravity on the brane was introduced in [16] where the foundation of model based on a geometrical hypothesis. In [16] the authors investigated the most general geometrical scenarios in which a brane-world program compatible with the hypotheses of embedding, confinement and the existence of quantum states can be implemented and showed that their analysis is independent of any previous choice of geometry, topology, number of dimensions and signature for the bulk There have also been arguments concerning the uniqueness of the junction conditions. Indeed, other forms of junction conditions exist, so that different conditions may lead to different physical results [17. Furthermore, these conditions cannot be used when more than one non-compact extra dimension is involved.

In this paper, we follow [14, 15] and consider an $m$-dimensional bulk space without imposing any junction condition. Instead we used confining potential approach that localized gravitons according to the equation (41) on the brane. Finally we argue that the value of mass of graviton that in this model is quantized.

\section{The model}

In this section we present a brief review of the model proposed in [14, 15. Consider the background manifold $\bar{V}_{4}$ isometrically embedded in a pseudo-Riemannian manifold $V_{m}$ by the map $\mathcal{Y}: \bar{V}_{4} \rightarrow V_{m}$ such that

$$
\mathcal{G}_{A B} \mathcal{Y}_{, \mu}^{A} \mathcal{Y}_{, \nu}^{B}=\bar{g}_{\mu \nu}, \quad \mathcal{G}_{A B} \mathcal{Y}_{, \mu}^{A} \mathcal{N}_{a}^{B}=0, \quad \mathcal{G}_{A B} \mathcal{N}_{a}^{A} \mathcal{N}_{b}^{B}=g_{a b}= \pm 1
$$

where $\mathcal{G}_{A B}\left(\bar{g}_{\mu \nu}\right)$ is the metric of the bulk (brane) space $V_{m}\left(\bar{V}_{4}\right)$ in arbitrary coordinates, $\left\{\mathcal{Y}^{A}\right\}\left(\left\{x^{\mu}\right\}\right)$ is the basis of the bulk (brane) and $\mathcal{N}_{a}^{A}$ are $(m-4)$ normal unite vectors, orthogonal to the brane. The deformation of $\bar{V}_{4}$ in a sufficiently small neighborhood of the brane along an arbitrary transverse direction $\zeta$ is given by

$$
\mathcal{Z}^{A}\left(x^{\mu}, \xi^{a}\right)=\mathcal{Y}^{A}+\left(\mathcal{L}_{\zeta} \mathcal{Y}\right)^{A},
$$

where $\mathcal{L}$ represents the Lie derivative. By choosing $\zeta^{a}$ orthogonal to the brane, we ensure gauge independency [14] and have deformations of the embedding along a single orthogonal extra direction $\overline{\mathcal{N}}_{a}$ giving local coordinates of the deformed brane as

$$
\mathcal{Z}_{, \mu}^{A}\left(x^{\nu}, \xi^{a}\right)=\mathcal{Y}_{, \mu}^{A}+\xi^{a} \overline{\mathcal{N}}_{a, \mu}^{A}\left(x^{\nu}\right),
$$

where $\xi^{a}(a=1,2, \ldots, m-4)$ is a small parameter along $\mathcal{N}_{a}^{A}$ that parameterizes the extra noncompact dimensions. One can see from equation (2) that since the vectors $\overline{\mathcal{N}}^{A}$ depend only on the local coordinates $x^{\mu}$, they do not propagate along the extra dimensions

$$
\mathcal{N}_{a}^{A}\left(x^{\mu}\right)=\overline{\mathcal{N}}_{a}^{A}+\xi^{b}\left[\overline{\mathcal{N}}_{b}, \overline{\mathcal{N}}_{a}\right]^{A}=\overline{\mathcal{N}}_{a}^{A} .
$$

The above assumptions lead to the embedding equations of the deformed geometry

$$
\mathcal{G}_{\mu \nu}=\mathcal{G}_{A B} \mathcal{Z}^{A}{ }_{, \mu} \mathcal{Z}^{B}{ }_{, \nu}, \quad \mathcal{G}_{\mu a}=\mathcal{G}_{A B} \mathcal{Z}^{A}{ }_{, \mu} \mathcal{N}^{B}{ }_{a}, \quad \mathcal{G}_{a b}=\mathcal{G}_{A B} \mathcal{N}^{A}{ }_{a} \mathcal{N}^{B}{ }_{b} .
$$

If we set $\mathcal{N}_{a}^{A}=\delta_{a}^{A}$, the metric of the bulk space can be written in the following matrix form

$$
\mathcal{G}_{A B}=\left(\begin{array}{cc}
g_{\mu \nu}+A_{\mu c} A_{\nu}^{c} & A_{\mu a} \\
A_{\nu b} & g_{a b}
\end{array}\right)
$$


where

$$
g_{\mu \nu}=\bar{g}_{\mu \nu}-2 \xi^{a} \bar{K}_{\mu \nu a}+\xi^{a} \xi^{b} \bar{g}^{\alpha \beta} \bar{K}_{\mu \alpha a} \bar{K}_{\nu \beta b}
$$

is the metric of the deformed brane, so that

$$
\bar{K}_{\mu \nu a}=-\mathcal{G}_{A B} \mathcal{Y}_{, \mu}^{A} \mathcal{N}_{a ; \nu}^{B},
$$

represents the extrinsic curvature of the original brane (second fundamental form). Also, we use the notation $A_{\mu c}=\xi^{d} A_{\mu c d}$, where

$$
A_{\mu c d}=\mathcal{G}_{A B} \mathcal{N}_{d ; \mu}^{A} \mathcal{N}_{c}^{B}=\bar{A}_{\mu c d}
$$

represents the twisting vector fields (the normal fundamental form). Any fixed $\xi^{a}$ signifies a new deformed geometry, enabling us to define an extrinsic curvature similar to the original one by

$$
\widetilde{K}_{\mu \nu a}=-\mathcal{G}_{A B} \mathcal{Z}^{A}{ }_{, \mu} \mathcal{N}^{B}{ }_{a ; \nu}=\bar{K}_{\mu \nu a}-\xi^{b}\left(\bar{K}_{\mu \gamma a} \bar{K}_{\nu b}^{\gamma}+A_{\mu c a} A_{b \nu}^{c}\right) .
$$

Note that definitions (7) and (10) require

$$
\widetilde{K}_{\mu \nu a}=-\frac{1}{2} \frac{\partial \mathcal{G}_{\mu \nu}}{\partial \xi^{a}} .
$$

In geometric language, the presence of gauge fields $A_{\mu a}$ tilts the embedded family of sub-manifolds with respect to the normal vector $\mathcal{N}^{A}$. According to our construction, the original brane is orthogonal to the normal vector $\mathcal{N}^{A}$. However, equation (5) shows that this is not true for the deformed geometry. Let us change the embedding coordinates and set

$$
\mathcal{X}_{, \mu}^{A}=\mathcal{Z}_{, \mu}^{A}-g^{a b} \mathcal{N}_{a}^{A} A_{b \mu} .
$$

The coordinates $\mathcal{X}^{A}$ describe a new family of embedded manifolds whose members are always orthogonal to $\mathcal{N}^{A}$. In this coordinates the embedding equations of the deformed brane is similar to the original one, described by equations (11), so that $\mathcal{Y}^{A}$ is replaced by $\mathcal{X}^{A}$. This new embedding of the local coordinates are suitable for obtaining induced Einstein field equations on the brane. The extrinsic curvature of a deformed brane then becomes

$$
K_{\mu \nu a}=-\mathcal{G}_{A B} \mathcal{X}_{, \mu}^{A} \mathcal{N}_{a ; \nu}^{B}=\bar{K}_{\mu \nu a}-\xi^{b} \bar{K}_{\mu \gamma a} \bar{K}_{\nu b}^{\gamma}=-\frac{1}{2} \frac{\partial g_{\mu \nu}}{\partial \xi^{a}},
$$

which is the generalized York relation and shows how the extrinsic curvature propagates as a result of the propagation of the metric in the direction of extra dimensions. Now, let us write the Einstein equations in the bulk space as

$$
G_{A B}^{(b)}+\Lambda^{(b)} \mathcal{G}_{A B}=-\alpha^{*} S_{A B}
$$

where $\alpha^{*}=\frac{1}{M_{*}^{m-2}}\left(M_{*}\right.$ is the fundamental scale of energy in the bulk space), $\Lambda^{(b)}$ is the cosmological constant of the bulk and $S_{A B}$ consists of two parts

$$
S_{A B}=T_{A B}+\frac{1}{2} \mathcal{V} \mathcal{G}_{\mathcal{A B}}
$$

where $T_{A B}$ is the energy-momentum tensor of the matter confined to the brane through the action of the confining potential $\mathcal{V}$ [14, 15]. Using the Einstein equations (14) and Gauss-Codazzi equations, the tangent component of equation (14) becomes (gravi-tensor equation) 15.

$$
\begin{aligned}
& G_{\mu \nu}-\left(\frac{m-7}{m-1}\right) \Lambda^{(b)} g_{\mu \nu}=Q_{\mu \nu}-\mathcal{E}_{\mu \nu}-\frac{2}{m-2} \alpha^{*} S_{\mu \nu} \\
& -\left(\frac{m-3}{m-2}\right) \alpha^{*} g^{a b} S_{a b} g_{\mu \nu}+\frac{(m-4)(m-3)}{(m-2)(m-1)} \alpha^{*} S g_{\mu \nu},
\end{aligned}
$$

where $\mathcal{E}_{\mu \nu}=g^{a b} \mathcal{C}_{A B C D} \mathcal{N}_{a}^{A} \mathcal{X}_{\mu}^{B} \mathcal{X}_{\nu}^{C} \mathcal{N}_{b}^{D}$ is the electric part of Weyl tensor of the bulk space $\mathcal{C}_{A B C D}$, and

$$
Q_{\mu \nu}=g^{a b}\left(K_{a \mu \gamma} K_{b \nu}^{\gamma}-K_{a} K_{b \mu \nu}\right)-\frac{1}{2}\left(K_{a \alpha \beta} K^{a \alpha \beta}-K^{a} K_{a}\right) g_{\mu \nu}
$$

On the other hand, again from (14), the trace of Codazzi's equation gives the gravi-vector equation

$$
K_{a \gamma ; \delta}^{\delta}-K_{a, \gamma}-A_{b a \gamma} K^{b}+A_{b a \delta} K^{b \delta}+B_{a \gamma}=\frac{3(m-4)}{m-2} \alpha^{*} S_{a \gamma},
$$


where

$$
B_{a \gamma}=g^{m n} C_{A B C D} \mathcal{N}_{m}^{A} \mathcal{N}_{a}^{B} \mathcal{X}_{, \gamma}^{C} \mathcal{N}_{n}^{D} .
$$

Finally, gravi-scalar equation is given by 15

$$
\alpha^{*}\left[\frac{m-5}{m-1} S-g^{m n} S_{m n}\right] g_{a b}=\frac{m-2}{6}(Q+R+W) g_{a b}-\frac{4}{m-1} \Lambda^{(b)} g_{a b},
$$

where

$$
W=g^{a b} g^{m n} C_{A B C D} \mathcal{N}_{m}^{A} \mathcal{N}_{b}^{B} \mathcal{N}_{n}^{C} \mathcal{N}_{a}^{D} .
$$

Equations (16)-(21) represents the Einstein field equations on the bulk space near the original brane, represented in the Gaussian coordinates. The confinement hypotheses is applied as an effect of confinement potential on the original brane, and can be implemented simply as $[15$.

$$
\alpha \tau_{\mu \nu}=\frac{2}{(m-2)} \alpha^{*} \bar{T}_{\mu \nu}, \quad \bar{T}_{\mu a}=0, \quad \bar{T}_{a b}=0,
$$

where $\alpha$ is the scale of energy on the brane. Now, the induced Einstein field equation on the original brane can be written

$$
G_{\mu \nu}+\Lambda g_{\mu \nu}=-\alpha \tau_{\mu \nu}+\frac{(m-4)(m-3)}{2(m-1)} \alpha \tau g_{\mu \nu}+Q_{\mu \nu}-\mathcal{E}_{\mu \nu},
$$

where $\Lambda=-\frac{m-7}{m-1} \Lambda^{(b)}$. As was mentioned before, $Q_{\mu \nu}$ is a conserved quantity which according to [13] may be considered as an energy-momentum tensor of a dark energy fluid representing the $x$-matter, the more common phrase being "X-Cold-Dark Matter". This matter has the most general form of the equation of state which is characterized by the following conditions [18: first it violates the strong energy condition at the present epoch for $\omega_{x}<-1 / 3$ where $p_{x}=\omega_{x} \rho_{x}$, second, it is locally stable i.e. $c_{s}^{2}=\delta p_{x} / \delta \rho_{x} \geq 0$, and third, causality holds good, that is $c_{s} \leq 1$. Ultimately, we have three different types of "matter" on the right hand side of equation (16), namely, ordinary confined conserved matter represented by $\tau_{\mu \nu}$, the XCDM matter represented by $Q_{\mu \nu}$ and finally, the Weyl matter $\mathcal{E}_{\mu \nu}$.

\section{Linearization}

To discuss linearized gravity in this approach, consider the linear expansion of the bulk metric around the $m D$ background spacetime

$$
\mathcal{G}_{A B}=\hat{\mathcal{G}}_{A B}+h_{A B} .
$$

where $\hat{\mathcal{G}}_{A B}$ is the bulk space background metric and $h_{A B}$ is the deformation of the metric. Let us perturb the Einstein equations of motion (14) around the $\hat{\mathcal{G}}_{A B}$. The resulting field equation is

$$
\delta G_{A B}^{(b)}=\left(\frac{1}{2} \alpha^{*} \mathcal{V}-\Lambda^{(b)}\right) h_{A B}
$$

where $\delta G_{A B}^{(b)}$ is the perturbation of the bulk space Einstein tensor which is given by [19]

$$
\delta G_{A B}^{(b)}=\tilde{G}_{A B}^{(a)}+\Psi_{A B},
$$

where

$$
\tilde{G}_{A B}^{(a)}=\frac{1}{2}\left(\square h_{A B}-h_{C(A ; B)}{ }^{; C}+h_{; A B}-\mathcal{G}_{A B}\left(\square h-h_{; C D}^{C D}\right)\right),
$$

and

$$
\Psi_{A B}=\frac{1}{2}\left(\hat{\mathcal{G}}_{A B} \hat{\mathcal{R}}^{C D} h_{C D}-\hat{\mathcal{R}} h_{A B}\right) .
$$

where $\hat{\mathcal{R}}^{C D}$ and $\hat{\mathcal{R}}$ are associated with $\hat{\mathcal{G}}_{A B}$. It is straightforward to show that the linear approximation of Einstein field equation in the bulk space, in the harmonic gauge $h_{A}^{A}=0$ and $h_{B ; A}^{A}=0$, for de Sitter brane is

$$
\square h_{A B}-\hat{\mathcal{G}}_{A B} \hat{\mathcal{R}}^{C D} h_{C D}-\hat{\mathcal{R}} h_{A B}=\alpha^{*} \mathcal{V} h_{A B}-2 \Lambda^{(b)} h_{A B} .
$$


Inserting extra conditions $h_{a b}=h_{a \mu}=0$ [20], we can now write the induced linearized field equation on the perturbed brane

$$
\square h_{\mu \nu}+\frac{2}{m-2}\left(\alpha^{*} \mathcal{V}-\frac{m-1}{m-7} \Lambda\right) h_{\mu \nu}=0 .
$$

To obtain field equations on the original brane which according to [14] describe the physical $4 D$ universe in large scales, we expand the d'Alembertian and confining potential around the original geometric configuration. In general we can expand the confining potential in terms of extra dimensions $\xi^{a}$

$$
\mathcal{V}=\mathcal{V}_{0}+\xi^{a} \mathcal{V}_{, a}+\frac{1}{2 !} \xi^{a} \xi^{b} \mathcal{V}_{, a b}+\mathcal{O}\left(\xi^{3}\right)
$$

where $\mathcal{V}_{0}$ is a constant that can be absorbed in cosmological constant. To preserving the symmetries of the induced gauge fields, we assume that $\mathcal{V}_{, a b}=\omega^{2} g_{a b}[14$. On the other hand, to cancel the higher order expansion in the equation (31), we consider $\omega$ to be much larger than the inverse of the scale of curvatures $\rho^{-1}$ on the original brane, or more specifically $\omega \gg \rho^{-2}[21]$. This means the effective size of extra dimensions through which the graviton can propagate, is more smaller than the distance of horizon denoted by $\rho$. Now, we adsorb the scale of $\omega$ into a small dimensionless parameter $\sigma$, that is $\omega \rightarrow \omega / \sigma$, so that $\omega$ becomes of the same order as $\rho^{-2}$. In fact, the "squeezing" parameter $\sigma$ plays the role of natural perturbation parameter. To identify $\mathcal{V}_{, a}$, let us attend to the conservation equation of $S^{A B}$ in the bulk space. From Einstein field equations (14) we have

$$
S_{; A}^{A B}=0 .
$$

Now with an eye on the confinement hypotheses (22), we have for the extra-extra components of equation (32) on the original brane

$$
\Gamma_{\mu c}^{\mu} T^{c b}+\Gamma_{\mu \nu}^{b} T^{\mu \nu}+\Gamma_{a c}^{a} T^{c b}+\frac{1}{2} \mathcal{V}^{, b}=0
$$

where $\Gamma$ denotes the connection coefficients on the bulk space. Using relation of connection coefficients on the bulk with induced quantities obtained in [22, equation (33) becomes

$$
\bar{K}_{\mu \nu}^{a} \bar{T}^{\mu \nu}=\mathcal{V}^{, a}
$$

Hence confining dictates

$$
\mathcal{V}^{, a}=\bar{K}_{\mu \nu}^{a} \bar{T}^{\mu \nu}=\frac{m-2}{2} \frac{\alpha}{\alpha^{*}} \bar{K}_{\mu \nu}^{a} \tau^{\mu \nu} .
$$

Also, using the explicit form of the Gaussian metric (12) the d'Alembertian operator on the deformed brane appeared in equation (30) can be written as

$$
\square=-\frac{1}{|g|^{1 / 4}} \partial_{a}|g|^{1 / 2} \partial^{a} \frac{1}{|g|^{1 / 4}}-\frac{1}{|\bar{g}|^{1 / 4}|g|^{1 / 4}} \partial_{\mu} g^{\mu \nu}|g|^{1 / 4} \partial_{\nu} \frac{|\bar{g}|^{1 / 4}}{|g|^{1 / 4}} .
$$

Since $\sigma$ is the perturbation parameter, by changing the extra coordinates as $\xi^{a} \rightarrow \sigma^{1 / 2} \xi^{a}$, the d'Alembertian and confining potential can be expanded in powers of $\sigma$, leading to

$$
\square=\frac{1}{\sigma} \square^{(0)}+\square^{(1)}+\cdots,
$$

and

$$
\mathcal{V}=\frac{m-2}{2} \frac{\alpha}{\alpha^{*}} \sigma^{\frac{1}{2}} \xi^{a} \bar{K}_{a \mu \nu} \tau^{\mu \nu}+\frac{1}{2 !} \frac{\omega^{2}}{\sigma} g_{a b} \xi^{a} \xi^{b}+\mathcal{O}\left(\sigma^{\frac{3}{2}}\right)
$$

where

$$
\square^{(0)}=-g^{a b} \partial_{a} \partial_{b}
$$

and

$$
\square^{(1)}=-\frac{1}{|\bar{g}|^{1 / 2}} \partial_{\mu} \bar{g}^{\mu \nu}|\bar{g}|^{1 / 2} \partial_{\nu}+\frac{1}{4} \bar{g}^{\mu \nu} \bar{g}^{\alpha \beta}\left(g_{a b} \bar{K}_{\mu \nu}^{a} \bar{K}_{\alpha \beta}^{b}-2 g_{a b} \bar{K}_{\mu \alpha}^{a} \bar{K}_{\nu \beta}^{b}\right)
$$




\section{Wave functions and mass quantization}

Now using the equations (29)-(40) and the ansatz that $h_{\mu \nu}$ can be decompose as $h_{\mu \nu}\left(x^{\mu}, \xi\right)=\Sigma_{\beta} h_{\mu \nu}^{(\beta)}\left(x^{\mu}\right) \Psi_{n}^{(\beta)}(\xi)$, where the index $\beta$ runs over any degeneracy that exists in the spectrum of the normal degrees of freedom, then the wave equation (30) splits to the following two wave equations, normal and tangent to the brane respectively

$$
\left(\square^{(0)}+\frac{\alpha^{*}}{m-2} \omega^{2} g_{a b} \xi^{a} \xi^{b}\right) \Psi_{n}^{(\beta)}(\xi)=\sigma E_{n} \Psi_{n}^{(\beta)}(\xi),
$$

and

$$
\left(-\frac{1}{|\bar{g}|^{1 / 2}} \partial_{\mu} \bar{g}^{\mu \nu}|\bar{g}|^{1 / 2} \partial_{\nu}+\mathcal{D}-E_{n}-\frac{2(m-1)}{(m-7)(m-2)} \Lambda\right) h_{\mu \nu}^{(\beta)}\left(x^{\mu}\right)=0
$$

where

$$
\mathcal{D}=\frac{1}{4}\left(g^{a b} \bar{K}_{a} \bar{K}_{b}-2 \bar{K}_{\alpha \beta a} \bar{K}^{\alpha \beta a}\right),
$$

and

$$
\sigma E_{n}=\left(\frac{2 \alpha^{*}}{m-2}\right)^{\frac{1}{2}} \omega \sum_{n=n_{1}}^{n_{m-4}} \epsilon_{n}\left(n+\frac{1}{2}\right),
$$

is the eigenfunction corresponding to the normal degrees of freedom, describing $m-4$ uncoupled harmonic oscillators. We know from quantum mechanics that for harmonic oscillator only probability of even modes around the minimum of potential is maximum, thus in this model only even modes of wave functions are localized on the brane $\left(\xi^{a}=0\right)$, in contrast to the usual brane models that only zero mode of wave equation is localized on the brane [23. It can be seen from equation (41), without confining potential $\mathcal{V}$ the equation (41) reduce to plane wave equation thus we can not localize gravity on the brane. Hence not only we need confining potential to localized test particles on the brane, but also it have the central role in confining of gravitons and the amount of graviton mass.

According to the [14] and [24], we assume $\alpha^{*} \omega^{2}=\frac{2}{\beta^{2}(m-2)} \Lambda^{2}$. Equations (42) and (44) show that the mass of graviton is quantized

$$
M^{2}=\frac{2(m-1)}{(m-7)(m-2)} \Lambda\left(1+\frac{1}{\sigma} \sum_{n=n_{1}}^{n_{m-4}} \epsilon_{n}\left(n+\frac{1}{2}\right)\right) .
$$

In equation (45) as $\sigma \rightarrow 0$, the mass becomes very large. On the other hand, if we change the coordinates according to $x^{\mu} \rightarrow \sqrt{\sigma} x^{\mu}$, then equation (44) reduces to the following form

$$
-\frac{1}{|\bar{g}|^{1 / 2}} \partial_{\mu} \bar{g}^{\mu \nu}|\bar{g}|^{1 / 2} \partial_{\nu} h_{\mu \nu}^{(\beta)}\left(x^{\mu}\right)-\sigma E_{n} h_{\mu \nu}^{(\beta)}=0 .
$$

The above equation shows that the redefined mass is given by

$$
\tilde{M}^{2}=\sigma E_{n}=\frac{2(m-1)}{(m-7)(m-2)} \Lambda \sum \epsilon_{n}\left(n+\frac{1}{2}\right) .
$$

Since our change of coordinates amounted to $x^{\mu} \rightarrow \sqrt{\sigma} x^{\mu}$, we relate this mass to the microworld. It is the mass of a quantum perturbation in a spacetime with very small curvature measured by the astrophysical value of $\Lambda$ as opposed to the mass sometimes inferred from the zero point or vacuum fields of particle interactions. As we noted above, the mass denoted by $M$ as a consequence of the large-scale gravitational effects and becomes very large according to $M=\tilde{M} / \sigma$. One may interpret this as having a parameter relating the large scale to that of the small. To have a feeling of $\sigma$, one may use the fundamental constants to construct it. We assume that our brane has a finite small thickness or width $l$. It also suggests to link the squeezing parameter $\sigma$, determined by the constraining potential, to the width of brane, that is

$$
\sigma=a l^{b}
$$

where $a$ is a dimensional constant so as to making $\sigma$ dimensionless so that one may use fundamental constants to determine the constants $a$ and $b$. If we set $a=L^{-b}$ were $L=\sqrt{\Lambda / 3}$ is the intrinsic length scale of the 
universe and if we set $b=2$ (this identification required from our rescaling property of coordinates), then we have

$$
\sigma=\left(\frac{l}{L}\right)^{2}
$$

According to the present experimental data in colliders, the value of $l$ should be less or of order of $T e V^{-1} \sim$ $10^{-17} \mathrm{~cm}$. If the brane width is $l$, it means that brane localized particles probe this length scale across the brane and therefor the observer cannot measure the distance on the brane to a better accuracy than $l$. Hence we have

$$
\sigma \sim\left(\frac{10^{-17} \mathrm{~cm}}{10^{28} \mathrm{~cm}}\right)^{2}=10^{-90} .
$$

Now, inserting the appropriate units into equation (47), we obtain the fundamental mass $\tilde{M}$

$$
\tilde{M}_{0}=\frac{\hbar}{c} \Lambda^{\frac{1}{2}} \sim 10^{-65} \mathrm{gr}
$$

Having made an estimate for $\sigma$, we can now obtain an order of magnitude for $M_{0}$

$$
M_{0} \sim\left(\frac{L}{l}\right)^{2} \tilde{M}_{0} \sim 10^{25} \mathrm{gr}
$$

On the other hand, the observational data constrains the brane width to be in order of planck length, see [24] and references therein. In this case we have [14]

$$
\sigma=\left(\frac{l_{P l}}{L}\right)^{2}=\frac{c^{3}}{\hbar G|\Lambda|} \sim 10^{-120}
$$

and consequently

$$
M_{0}=\frac{\Lambda c^{4}}{G} \frac{1}{|\Lambda|^{3 / 2}} \frac{1}{c^{2}}=\frac{c^{2}}{G|\Lambda|^{1 / 2}} \sim 10^{56} \mathrm{gr},
$$

where $l_{P l}$ is the Planck length. Let us ask about the meaning that one should attribute to this $M$. The second equality in (54) contains three separate factors. The first one represents the energy density generated by $\Lambda$; the second term $|\Lambda|^{-3 / 2}$ is the total volume of the universe restricted to its horizon and the last term is just to convert the total energy into a mass. Thus we can interpret it as the total mass of all existing gravitons in the observed universe. As was noted, the squeezing parameter $\sigma$ was introduced as a consequence of requiring a test particle to be confined to our brane. This parameter, however, opens up an opportunity in the way of observing our universe from two different angles, namely, either looking at our universe in its entirety, that is, at its large-scale structure, or do the opposite, i.e. observing it from small scales. The parameter $\sigma$ provides us with the tool necessary to achieve this. It suffices to define the change of variable introduced earlier, that is $x^{\prime \mu}=\sqrt{\sigma} x^{\mu}$. This relation connects two very different scales: small and large. Now the question of the disparity between the values of the cosmological constant in cosmology and particle physics reduces to its measurement from two different scales. If we look at it from the large-scale point of view, we measure its astrophysical value. On the other hand, if one looks at its value from very small scales, it turns out to be related to that of $\Lambda$ through the relation $\tilde{\Lambda}=\sigma^{-2} \Lambda$ which is 240 orders of magnitude larger than its astrophysical value. The unusually large order of magnitude should not alarm the reader for if we had used the Planck mass in equation (52) instead of $M_{0}$, we would have obtained the usual value for the order of magnitude, that is 120 . The above discussion leads us to the conclusion that the vast difference between the values of the cosmological constant simply stems from our measurements at two vastly different scales. Note that in $4 D$ generally covariant theory the mass is a scalar and is invariant under any coordinate transformation. On the other hand, in this paper, we introduced first a higerdimensional bulk space where the physical laws are assumed to be covariant under general coordinate transformations and local redefinitions of reference frames.

$$
\mathcal{Z}^{A} \rightarrow \mathcal{Z}^{\prime A}(\mathcal{Z}), \quad E_{I}^{A} \rightarrow \Lambda_{I}^{B}(\mathcal{Z}) E_{B}^{A}
$$

so that $\mathcal{G}_{A B} E_{I}^{A} E_{J}^{B}=\eta_{I J}$. Also our assumption in the reduction of dimensions of the bulk space to the brane is that the brane is parameterized by coordinates $x^{\mu}$ and reference frames are made up 4 reference vectors $e_{\alpha}^{\mu}, \quad \alpha=0, \ldots, 3.4 D$ physical laws are covariant under general coordinate transformations $x^{\mu} \rightarrow x^{\prime \mu}(x)$, see for more details [25]. The existence of a specified brane that we are living in, dictates the broken of general covariance in the bulk space [25]. Consequently the re-scaling of graviton mass is a direct result of the 
breakdown of general covariance in the bulk space. For a test particles that are exactly confined to the brane, the influence of $4 D$ general covariance takes the mass of them invariant, but the graviton can be fluctuate in the bulk space and consequently detects the breakdown of $m D$ general covariance.

To make progress in our understanding of braneworlds, linear gravitational waves can also be most helpful. Linear gravitational waves in the bulk space are supposed to propagate both in the bulk and in the brane-world. One such propagation was considered in [26, with the surprising result that the the bulk wave interfered with the binary pulsar PSR1913+16 27], producing a strong contrast with the Hulse-Taylor estimates on orbit decay. In higher-dimensional theories where there are more than one extra dimensions the above result is not useable where in these models there are several gravi-scalars which may all contribute a similar amount to the emission of gravity waves [26]. The major exception is that in warped models with non-compact extra dimension such as Randall-Sundrum II model and our model, where the scalar gravity mode is not normalizable, this discussion

dose not apply. Also in this paper we have studied a non-compact model without junction conditions and therefor the calculations are not extendable to our model.

\section{Conclusions}

In this paper we have studied a perturbed brane world model in which the matter is confined to the brane through the action of a confining potential, rendering the use of any junction condition redundant. In this approach, the mass of graviton is quantized and the cosmological constant problem can be addressed as the measurement problem. Also the spectrum of graviton is discrete with localized even modes. The quantization of mass in fact is the result of fluctuation of graviton around the original non-deformed brane which is in agreement with results obtained in Induced Matter Theory of P. S. Wesson [28] and confined quantized test particles in the branes [14].

\section{Acknowledgements}

The authors thank Prof. Sepangi for reading the manuscript.

\section{References}

[1] S. Perlmutter et al. [Supernova Cosmology Project Collaboration], Astrophys. J. 517 (1999) 565.

[2] L. Randall and R. Sundrum, Phys. Rev. Lett. 83 (1999) 4690 .

[3] J. Madlacena, Adv. Theor. Math. Phys. 2 (1998) 231.

[4] E. Witten, Adv. Theor. Math. Phys. 2 (1998) 253.

[5] S. S. Gubser, I. R. Klebanov and A. M. Polyakov, Phys. lett. B 428 (1998) 105.

M. Ito, Phys. lett. B 528 (2002) 269 .

[6] W. Israel, Nuovo Cimento B 44 (1966) 1.

[7] I. Brevik, K. Ghoroku, S. D. Odintsov and M. Yahiro, Phys. Rev. D 66 (2002) 064016.

[8] M. Chaichian and A. B. Kobakhidze, Phys. Lett. B 488 (2000) 117.

[9] R. Durrer and P. Kocian, Class. Quant. Grav. 21 (2004) 2127.

[10] J. L. Feng, A. Rajaraman and F. Takayama, Phys. Rev. D 68 (2003) 085018.

[11] V. A. Rubakov and M. E. Shaposhnikov, Phys. Lett. B 125 (1983) 136 .

[12] M. D. Maia, E. M. Monte and J. M. F. Maia, Phys. Lett. B 585 (2004) 11.

[13] M. D. Maia, E. M. Monte, J. M. F. Maia and J. S. Alcaniz, Class. Quant. Grav. 22 (2005) 1623.

[14] S. Jalazadeh and H. R. Sepangi, Class. Quant. Grav. 22 (2005) 2035. 
[15] M. Heydari-fard, M. Shirazi, S. jalalzadeh and H. R. Sepangi, Phys. Lett. B 640 (2006) 1 , M. Heydari-Fard and H. R. Sepangi, Phys. Rev. D 76 (2007) 104009,

M. Heydari-Fard, H. Razmi and H. R. Sepangi, Phys. Rev. D 76 (2007) 066002,

M. Heydari-Fard and H. R. Sepangi, Phys. Lett. B 649 (2007) 1,

M. Heydari-Fard and H. R. Sepangi, Phys.Rev. D 75 (2007) 064010.

[16] M. D. Maia and Edmundo M. Monte Phys. Lett. A 297 (2002) 9.

[17] R. A. Battye and B. Carter, Phys. Lett. B 509 (2001) 331.

[18] M. S. Turner and M. White, Phys. Rev. D 56 (1997) R4439, Chiba T. Sugiyama and T. Nakamura, Mon. Not. R. Astron. Soc. 289 (1997) L5 .

[19] M. Novello and R.P. Neves, Class. Quant. Grav. 20 (2003) L67 .

[20] P. Singh and N. Dadhich, Mod. Phys. Lett. A 18 (2003) 983.

[21] P. C. Schuster and R. L. Jaffe Ann. Phys. 307 (2003) 132.

[22] S. Jalalzadeh, B. Vakili, F. Ahmadi and H. R. Sepangi, Class. Quant. Grav. 23 (2006) 6015.

[23] M. Ito, Europhys. Lett. 64 (2003) 295.

[24] M. Maziashvili, arxiv: hep-ph/0607123.

[25] P. Maraner and J. K. Pachos, Annals of Physics 323 (2008) 2044, arXiv:0704.2076.

[26] R. Durrer and P. Kocian, Class. Quant. Grav. 21 (2004) 2127.

[27] J. H. Taylor, Rev. Mod. Phys. 66 (1994) 711; For an up to date review see C.M. Will, Living Rev. Relativity 4 (2001) 4: cited on April 2003, http://wwwlivingreviews.org/Articles/Volume4/2001-4will/

[28] P. S. Wesson, Mod. Phys. Lett. A 19 (2004) 1995. 